\title{
Repercussões Perinatais do Oligoidrâmnio sem Ruptura Prematura das Membranas Detectado até a 26a Semana de Gravidez
}

\author{
Perinatal Outcomes of Oligohydramnios without Premature Rupture of \\ Membranes Detected until the 26th Week of Pregnancy \\ Germana Zélia Gomes Bastos, Régis Oquendo Nogueira, Carlos Augusto Alencar Júnior
}

\section{RESUMO}

\begin{abstract}
Objetivo: avaliar as repercussões perinatais da oligoidramnia não decorrente da ruptura prematura das membranas (RPM), diagnosticada até a $26^{a}$ semana de gravidez.

Métodos: análise retrospectiva dos casos de oligoidramnia ocorridos entre janeiro de 1994 e dezembro de 2000 , diagnosticados até a $26^{a}$ semana de gravidez, empregando como critério índice de líquido amniótico igual ou inferior a $5 \mathrm{~cm}$. Após o diagnóstico, as grávidas foram acompanhadas com exames ecográficos seriados, atentando-se para a manutenção do quadro. Havendo regressão da oligoidramnia, as pacientes permaneceram na pesquisa. As pacientes com RPM, as com óbito fetal ao primeiro exame e as que tiveram parto em outra instituição foram excluídas. Em relação às grávidas interessou-nos, especialmente, a presença de doenças e complicações obstétricas associadas. Quanto ao recém-nascido, avaliaram-se, dentre outros, o peso ao nascimento, tempo de internação/óbito, ocorrência de óbito ou malformação.

Resultados: foram incluidos vinte e sete casos de oligoidramnia. Treze fetos apresentaram malformações, sendo oito do trato urinário, quatro do sistema nervoso central e um higroma cístico. Quatorze gestantes apresentaram complicações clínicas ou obstétricas, principalmente hipertensão (10 casos). Além destes, encontramos três casos de placenta prévia e um de tiroidopatia. Houve 13 óbitos fetais e 14 neonatais.

Conclusão: a oligoidramnia não decorrente da RPM, ocorrendo no segundo trimestre da gestação, independente da etiologia ou presença de anomalias congênitas, apresenta prognóstico sombrio e associou-se a resultado perinatal fatal em todos os casos.
\end{abstract}

PALAVRAS-CHAVE: Oligoidrâmnio. Resultado perinatal. Líquido amniótico. Malformações fetais. Óbito fetal.

\section{Introdução}

A oligoidramnia é caracterizada pela acentuada redução do volume de líquido amniótico. Sua incidência varia de acordo com a população estudada e os critérios diagnósticos utilizados, sendo estimada em torno de 0,5 a $5 \%{ }^{1}$. As principais causas determinantes são: ruptura prematura das

Maternidade Escola Assis Chateaubriand, Universidade Federal do Ceará

PIBIC - CNPq

Correspondência:

Germana Zélia Gomes Bastos

Rua Vilebaldo Aguiar, $545 \mathrm{Apt}^{\circ} 403$ - Cocó

60190-780 - Fortaleza - CE

Fone/Fax: (85) 265-6891 membranas (RPM), insuficiência placentária, anomalias congênitas, aneuploidias fetais e uso de medicações pela mãe durante a gravide $z^{2,3}$.

A hipoperfusão placentária é, provavelmente, a responsável pela associação com hipertensão arterial, tabagismo materno ${ }^{4}$, pós-maturida$\mathrm{de}^{5}$ e crescimento fetal restrito ${ }^{6}$.

A prevalência de anomalias congênitas varia entre 4,5 e $37 \%^{7-9}$. Merecem destaque especial as que comprometem o sistema urinário, principalmente agenesia, displasias e obstrução do trato urinário fetal ${ }^{4}$. Após a $24^{\mathrm{a}}$ semana gestacional, as anomalias renais já não representam as principais hipóteses etiológicas da oligoidramnia e outras causas devem ser procuradas. Vale destacar, também, no oligoidrâmnio precoce diagnosticado 
no segundo trimestre, em virtude do efeito mecânico sobre o feto, a presença de alterações faciais e esqueléticas ${ }^{10}$.

As aneuploidias estão presentes em 0,4 a $4,4 \%$ dos $\operatorname{casos}^{7-9}$. A associação entre restrição simétrica do crescimento fetal e oligoidramnia sugere possivel anormalidade do cariótipo ${ }^{6}$.

Alguns medicamentos também estão vinculados à diminuição do volume âmnico. Os inibidores da prostaglandina sintetase, como a indometacina, provocam oligoidrâmnio ao reduzir o fluxo vascular renal ${ }^{11}$. Os inibidores da enzima conversora da angiotensina, como o captopril, permitem sua instalação ao promover hipertensão fetal, insuficiência renal e anúria, ocasionalmente associadas à morte do feto ${ }^{12}$.

O diagnóstico clínico da oligoidramnia é tardio. Baseia-se na diminuição da altura uterina e fácil percepção das partes fetais à palpação obstétrica. A melhor forma de diagnóstico é por meio da avaliação ultra-sonográfica. Dentre as técnicas existentes destaca-se o índice de líquido amniótico (ILA), proposto como forma de analisar mais completamente o volume de líquido amniótico ${ }^{13}$. A oligoidramnia é definida quando o somatório é menor ou igual a $5,0 \mathrm{~cm}^{14}$.

É freqüente existir resultado perinatal desfavorável no oligoidrâmnio precoce, seja devido à causa determinante, seja pelas repercussões provocadas sobre o concepto pela diminuição extrema do líquido. Um dos fatores responsáveis pelos altos índices de morte é a hipoplasia pulmonar, decorrente, nos casos de oligoidramnia precoce, da compressão do tórax e abdome fetais, limitando a movimentação do diafragma ${ }^{10}$. A restrição do crescimento fetal, freqüentemente proporcional ao grau de oligoidramnia, está, também, relacionada ao incremento da mortalidade perinatal ${ }^{8}$.

A oligoidramnia, quando instalada até o $2^{\circ}$ trimestre, tem prognóstico reservado, e a mortalidade perinatal, relatada em trabalhos anteriores, varia entre 43 e 100\% ${ }^{15-17}$. Sugere-se, em virtude do desfecho freqüentemente desfavorável, a busca do fator etiológico.

Visando avaliar as repercussões perinatais da oligoidramnia no segundo trimestre, instalada e diagnosticada até a $26^{\mathrm{a}}$ semana gestacional, realizamos análise retrospectiva dos casos ocorridos em nossa instituição.

\section{Pacientes e Métodos}

Foram analisados retrospectivamente 58 casos de oligoidramnia diagnosticados no Serviço de Medicina Materno-Fetal da Maternidade Esco- la Assis Chateaubriand, no período de janeiro de 1994 a dezembro de 2000. Foram incluídos casos de oligoidramnia diagnosticados pela ultra-sonografia até a $26^{\mathrm{a}}$ semana de gravidez. Utilizou-se, como critério diagnóstico ecográfico, o ILA. Nesse método, o útero é dividido em quatro quadrantes, por duas linhas imaginárias perpendiculares que se cruzam ao nível da cicatriz umbilical. Em cada um deles mede-se o maior bolsão vertical de fluido, sendo o resultado do ILA a soma dos quatro valores obtidos. Considerou-se redução acentuada do fluido âmnico quando o somatório dos quatro bolsões de líquido, o maior de cada quadrante uterino, foi igual ou inferior a $5,0 \mathrm{~cm}^{13}$. Na avaliação ultra-sonográfica inicial definiu-se, também, a idade gestacional e a presença de malformações. Havendo discrepância superior a uma semana entre as idades gestacionais estimadas por última menstruação e ecografia, optou-se pela determinada pelo exame ultra-sonográfico.

Foram excluídas grávidas com idade gestacional superior a 26 semanas, as que tinham como etiologia da oligoidramnia a RPM e as expostas a qualquer tratamento invasivo previamente ao diagnóstico. Também foi motivo para saída da pesquisa a realização do parto em outro hospital. Pacientes com óbito fetal na primeira avaliação ecográfica não foram, igualmente, incluídas na pesquisa. As que apresentaram morte fetal nas avaliações posteriores foram mantidas.

As grávidas foram acompanhadas, quando possivel, com exames seriados até a resolução da gestação. Nestes casos, atentou-se para a manutenção da oligoidramnia. Quando houvesse regressão do quadro, as gestantes permaneciam na pesquisa.

Em relação às grávidas, interessaram-nos, ainda, a idade, o número de gestações e de partos e a presença de doenças clínicas e/ou obstétricas associadas. Quanto à resolução da gestação, avaliamos a idade gestacional em que ocorreu.

Quanto ao recém-nascido, obtivemos o peso ao nascimento, índices de Apgar de primeiro e quinto minutos, índice de Capurro somático, adequação do peso em relação à idade gestacional, tempo de internação e ocorrência de óbito fetal ou malformações. Todos os neonatos foram acompanhados até a alta ou óbito.

Foi aplicado protocolo de coleta de dados que continha perguntas sobre todas as informações supracitadas. Inicialmente, submetemos esse protocolo a um pré-teste com 10 prontuários para que fosse adequado aos objetivos do nosso estudo e, na etapa seguinte, foram aplicados aos demais casos já com as adaptações necessárias.

A análise dos dados foi realizada por meio do software Epi-Info 6.04. O trabalho foi aprovado pelo Comitê de Ética da instituição. 


\section{Resultados}

Foram analisados 58 casos de oligoidramnia em gestações com idade igual ou inferior a 26 semanas. Destes, vinte e sete foram incluídos no estudo. Foram excluídos vinte e cinco casos, por serem decorrentes de RPM, e outros seis, por não terem sido acompanhados até a resolução da gravidez em nosso serviço.

A idade materna foi, em média, de 27 anos, variando entre 15 e 44 anos. O número de gestações variou de 1 a 7 , com média de 2 gestações, sendo que 10 pacientes eram primigestas, 8 secundigestas e 9 multigestas. Em relação à paridade prévia, encontramos 13 nulíparas, 6 primíparas e $8 \mathrm{com}$ pelo menos dois partos. A paridade média foi de um parto.

Dentre as causas vinculadas às intercorrências clínicas maternas, a principal, sem dúvida, foi a hipertensão arterial, presente em 10 pacientes (37\%). Uma grávida era portadora de hipertiroidismo $(3,7 \%)$ e três apresentaram, além da oligoidramnia, inserção baixa de placenta $(11,1 \%)$.

As malformações, descritas na Tabela 1, estiveram presentes em 13 fetos $(48,1 \%)$, sendo 8 no trato urinário $(33,3 \%), 4$ no sistema nervoso central $(11,1 \%)$ e um caso de higroma cístico $(3,7 \%)$.

Tabela 1 - Malformações fetais evidenciadas em gestantes com oligoidrâmnio diagnosticado até a $26^{\mathrm{a}}$ semana gestacional.

\begin{tabular}{lc} 
Malformação fetal & Número \\
\hline Anencefalia & 1 \\
Hidrocefalia & 1 \\
Holoprosencefalia & 1 \\
Síndrome de Dandy-Walker & 1 \\
Higroma cístico & 1 \\
Agenesia renal bilateral & 1 \\
Rins multicísticos & 3 \\
Rins policísticos & 1 \\
Obstrução da válvula de uretra posterior & 2 \\
Cisto renal isolado & 1 \\
\hline
\end{tabular}

O ILA quando do diagnóstico variou de zero a $49 \mathrm{~mm}$. A anidramnia esteve presente em 14 casos. Em 20 pacientes foram realizados exames ultra-sonográficos seriados. Em 16 grávidas a oligoidramnia foi mantida e em 4 houve reversão do quadro, sendo que em dois o ILA tornou-se normal (ILA de $110 \mathrm{~mm}$ e $115 \mathrm{~mm}$ ). Entre os casos de anidramnia, 12 foram submetidos a exames seriados, sendo que em 8 a anidramnia foi mantida.

Apenas uma paciente fez uso de corticoterapia, na $26^{\mathrm{a}}$ semana gestacional. O par- to ocorreu no mesmo dia do diagnóstico. O recémnascido nasceu com 630 gramas, com Apgar de primeiro e quinto minuto de 6 e 8 , respectivamente, e foi a óbito em dois dias. Este recém-nascido apresentou, como intercorrências, síndrome do desconforto respiratório e plaquetopenia grave, evoluindo para parada cardiorrespiratória irreversivel.

Em relação ao parto, verificamos ter ocorrido entre a $21^{\text {a }}$ e a $38^{a}$ semana, com média de 27 semanas. Houve 13 óbitos fetais. Destes, cinco apresentaram malformações, previamente diagnosticadas no exame ultra-sonográfico. Quatorze conceptos foram a óbito após o nascimento, entre o primeiro e o décimo segundo dia de vida, com média de três dias, em virtude, principalmente, da prematuridade, encontrada em $96,3 \%$ dos casos, e da síndrome do desconforto respiratório, que esteve presente em todos os neonatos.

O maior peso ao nascimento foi de 3.180 gramas e o menor de 620 gramas, com média de 1.090 gramas. Dentre os óbitos neonatais, a média foi de 1.313 gramas. Nos casos de óbitos fetais esta média foi de 1000 gramas.

Apenas um recém-nascido recebeu alta de nosso serviço, sendo, entretanto, transferido, para o Hospital Infantil Albert Sabin, em nossa cidade. Tinha apresentado imagens sugestivas de cistos renais bilaterais ao exame ultra-sonográfico durante a gravidez. Ao nascimento, foi interrogada a possibilidade de trissomia do par 21. Evoluiu com grave insuficiência renal, permanecendo em nossa UTI neonatal por nove dias, tendo sido levantada a hipótese de válvula de uretra posterior, com hidronefrose bilateral. Foi transferido para realização de diálise peritoneal. Permaneceu no hospital de referência por dois dias, vindo a falecer por agravamento do quadro de insuficiência renal.

\section{Discussão}

A oligoidramnia está relacionada a significativo aumento da taxa de mortalidade perinatal se comparada à verificada em pacientes com volume de líquido amniótico normal ${ }^{10}$. A instalação do quadro até o segundo trimestre parece ter pior prognóstico, diminuindo significativamente a chance de sobrevivência do feto ${ }^{3,15,17}$. O péssimo resultado perinatal está vinculado ao incremento de malformações, especialmente renais, e à hipoplasia pulmonar, principalmente nos casos de oligoidramnia grave ${ }^{17}$.

Barss et al. ${ }^{15}$ não evidenciaram, em 12 casos de oligoidramnia diagnosticados entre a $13^{\mathrm{a}} \mathrm{e}$ a $25^{\mathrm{a}}$ semana, nenhum caso de sobrevida fetal, à semelhança do verificado em nosso estudo. Em sua série ocorreram quatro interrupções eletivas da 
gestação até a $22^{\mathrm{a}}$ semana, tendo-se evidenciado malformações em três destes conceptos. Nos oito casos restantes, cinco fetos apresentaram anomalias renais incompativeis com a vida, um recémnascido era prematuro extremo (22 semanas) e dois tiveram restrição acentuada do seu crescimento, sendo que um deles era portador de cariótipo triplóide. Os autores concluíram ser péssimo o prognóstico das gestações, mesmo na ausência de alterações da morfologia fetal.

Mercer et al. ${ }^{3}$ observaram oligoidramnia em 18 grávidas antes da $27^{\mathrm{a}}$ semana. O resultado foi igualmente desfavorável. Ocorreram seis abortos, cinco óbitos fetais e quatro neonatos com malformações incompativeis com a vida. Três partos aconteceram até a $26^{\mathrm{a}}$ semana, sem descrição, no trabalho, se houve sobrevivência neonatal.

Mercer e Brown ${ }^{16}$ também estudaram retrospectivamente 34 casos de oligoidramnia diagnosticados no $2^{\circ}$ trimestre. Nove conceptos eram portadores de alterações estruturais incompativeis com a vida. Além desses fetos, constataram 11 óbitos fetais, dos quais 10 foram de causa inexplicada e um por descolamento prematuro da placenta. Entre os 14 que nasceram vivos, ocorreram oito mortes neonatais. Diferente do referido pelo restante da literatura, conseguiram que seis crianças $(17,6 \%)$ nascessem a termo, com peso normal e índices de Apgar adequados, tanto de $1^{\circ}$ quanto de $5^{\circ}$ minuto. Por esse motivo, os autores concluíram que, apesar da acentuada morbidade e mortalidade perinatais, a resolução da gestação somente deva ser efetuada quando são diagnosticadas malformações ou anormalidades genéticas fetais.

Moore et al. ${ }^{17}$ avaliaram 62 casos diagnosticados entre a $13^{\mathrm{a}}$ e a $28^{\mathrm{a}}$ semana e observaram que, na presença de anidramnia ou oligoidramnia grave, definidas ecograficamente de maneira subjetiva pelo examinador, $88 \%$ dos conceptos apresentaram prognóstico letal. Vale destacar que 60\% dos recém-nascidos apresentaram hipoplasia pulmonar e 52\% anomalias congênitas maiores. Nos casos classificados como moderados ou leves o resultado foi sensivelmente melhor, com 11\% de óbitos, 6\% de hipoplasia pulmonar e 17\% de anomalias congênitas maiores.

Em nosso estudo o prognóstico perinatal letal foi semelhante ao relatado em outros estudos, da mesma forma como a observação de malformações incompativeis com a vida, destacando-se as decorrentes do trato urinário. Mesmo quando a etiologia foi materna, como no caso das mães hipertensas, o desfecho gestacional foi desfavorável.

Além disso, os resultados foram insatisfatórios mesmo quando houve normalização posterior do líquido amniótico, evidenciada em duas opor- tunidades, ou reversão da anidramnia, presente em quatro gestantes. Concluímos, portanto, que independente da etiologia da oligoidramnia, quando esta se manifesta até o $2^{\circ}$ trimestre da gestação, apresenta prognóstico extremamente sombrio. $\mathrm{O}$ achado do quadro em época precoce da gravidez deve ser comunicado aos pais e estes alertados para o péssimo resultado perinatal, independente, inclusive, da existência de malformações.

Somente uma paciente utilizou corticoterapia, mesmo assim sem o efeito desejado, já que foi usada no mesmo dia do parto. Não acreditamos, no entanto, que sua utilização sistemática tivesse modificado, ou viesse a modificar, o resultado da gravidez. Apesar disso, introduzimos, de rotina, a utilização da dexametasona, $12 \mathrm{mg}$ por via intramuscular, em dois dias seguidos, na tentativa de melhorar os nossos achados. Avaliaremos posteriormente os novos casos para definirmos se houve mudança no desfecho perinatal.

Chamou-nos a atenção o pequeno interesse evidenciado na literatura sobre o tema. Apenas um trabalho sobre oligoidramnia de instalação precoce, não decorrente da amniorrexe prematura, foi encontrado na última década. Embora achemos que o prognóstico seja reservado, os avanços tecnológicos, inclusive com a acentuada melhoria dos berçários, nos obrigam a continuar investigando tais casos, especialmente quando da ausência de malformações e anomalias cromossômicas.

\section{ABSTRACT}

Purpose: to evaluate the perinatal outcomes of pregnancies complicated by oligohydramnios, not due to premature rupture of membranes (PRM), diagnosed until the 26th week of gestation.

Patients and Methods: we analyzed retrospectively the cases of oligohydramnios that occurred from January 1994 to December 2000, and were diagnosed until the 26th week of gestation. Oligohydramnios was present when the amniotic fluid index was less or equal to $5.0 \mathrm{~cm}$. After diagnosis the patients were followed-up with serial ultrasound evaluation, with emphasis on the maintenance of the oligohydramnios state. When remission of the oligohydramnios occurred, patients remained in the study. Cases due to PRM, fetal death detected on the first examination and the women who gave birth in another institution were excluded from the study. Concerning the patients, the presence of clinical and obstetric diseases was investigated. As regards the newborns, we evaluated birth weight, time of admission/death, occurrence of death or malformations.

Results: twenty-seven cases of oligohydramnios were analyzed. Thirteen fetuses had congenital anomalies, and among them, eight had anomalies of the urinary tract, four of 
the nervous system and one had cystic hygroma. Fourteen patients had a clinical or an obstetric disease, mainly hypertension (10 cases). In addition, we found three cases of placenta previa and one case of thyropathy. There were thirteen fetal deaths and fourteen neonatal deaths.

Conclusion: oligohydramnios not due to PRM, occurring in the second trimester of gestation, independent of the etiology or the presence of congenital anomalies, was associated with a fatal perinatal result.

KEYWORDS: Oligohydramnios. Perinatal results. Amniotic fluid. Fetal malformations. Fetal death.

\section{Referências}

1. Lopes APBM. Sistema amniótico: oligoidrâmnio e polidrâmnio. In: Sociedade de Obstetrícia e Ginecologia de Minas Gerais. Ginecologia \& Obstetrícia: manual para o TEGO: título de especialista em ginecologia e obstetrícia. $2^{\text {a }}$ ed. Rio de Janeiro: Medsi; 2000. p.537.

2. Chamberlain PF, Manning FA, Morrison I, Harman CR, Lange IR. Ultrasound evaluation of the amniotic fluid volume. I. The relationship of marginal and decreased amniotic fluid volume to perinatal outcome. Am J Obstet Gynecol 1984; 150:245-9.

3. Mercer LJ, Brown LG, Petres RE, Messer RH. A survey of pregnancies complicated by decreased amniotic fluid. Am J Obstet Gynecol 1984; 149:355-61.

4. Tarari S, Treisser A, Renaud R. Oligohydramnios. Diagnosis. Etiology. Prognosis. J Gynecol Obstet Biol Reprod (Paris) 1987; 16:755-63.

5. Clement D, Schifrin BS, Kates RB. Acute oligohydramnios in postdate pregnancy. Am J Obstet Gynecol 1987; 157:884-6.

6. Nicolaides $\mathrm{KH}$, Rodeck $\mathrm{CH}$, Gosden CM. Rapid karyotyping in non-lethal fetal malformations. Lancet 1986; 1:283-7.
7. Nicolaides KH, Snijder RJM, Noble P. Cordocentesis in the study of growth-retard fetuses. In: Divon MY, editor. Abnormal Fetal Growth. $1^{\text {st }}$ ed. New York: Elsevier; 1991. p.166.

8. Hill LM, Breckle R, Wolfgram KR, O’Brien PC. Oligohydramnios: ultrasonically detected incidence and subsequent fetal outcome. Am J Obstet Gynecol 1985; 147:407-10.

9. Shipp TD, Bromley B, Pauker S, Frigoletto FD Jr, Benacerraf BR. Outcome of singleton pregnancies with severe oligohydramnios in the second and third trimesters. Ultrasound Obstet Gynecol 1996; 7:108-13.

10. Boyd RL, Carter SC. Polyhydramnios and oligohydramnios. eMed $\mathrm{J}$ [serial online] 2001; 2:1854. Available from: URL: http://www.emedicine.com/ ped/topic 1854.htm.

11.Hill LM, Lazebnik N, Many A. Effect of indomethacin on individual amniotic fluid indices in multiple gestations. J Ultrasound Med 1996; 15:395-9.

12.Benowitz NL. Agentes anti-hipertensivos. In: Katzung, BG, editor. Farmacologia Básica \& Clínica. $6^{\mathrm{a}}$ ed. Rio de Janeiro: Guanabara Koogan; 1998. p. 130.

13. Phelan JP, Ahn MU, Smith CV, Rutherford SE, Anderson E. Amniotic fluid index measurements during pregnancy. J Reprod Med 1987; 32:601-4.

14.Phelan JP, Smith CV, Broussard P, Small M. Amniotic fluid volume assessment with the four quadrant tecnique at 32-42 weeks' gestation. J Reprod Med 1987; 32:540-2.

15.Barss VA, Benacerraf BR, Frigolleto FD Jr. Second trimester oligohydramnios, a predictor of poor fetal outcome. Obstet Gynecol 1984; 64:608-10.

16. Mercer LJ, Brown LG. Fetal outcome with oligohydramnios in the second trimester. Obstet Gynecol 1986; 67:840-2.

17.Moore TR, Longo J, Leopold GR, Casola G, Gosink $\mathrm{BB}$. The reliability and predictive value of an amniotic fluid scoring system in severe second trimester oligohydramnios. Obstet Gynecol 1989; 73:739-42. 